\title{
Article
}

\section{Performance Evaluation of Topological Infrastructure in Internet-of-Things-Enabled Serious Games}

\author{
Shabir Ahmad, Faheem Khan and Taeg Keun Whangbo* \\ Department of I.T. Convergence Engineering, Gachon University, Sujeong-Gu, Seongnam-Si, Gyeonggi-Do, 461-701, \\ Korea \\ *Corresponding Author: Taeg Kuen Whangbo. Email: tkwhangbo@gachon.ac.kr \\ Received: 19 August 2021; Accepted: 22 September 2021
}

\begin{abstract}
Serious games have recently enticed many researchers due to their wide range of capabilities. A serious game is a mean of gaming for a serious job such as healthcare, education, and entertainment purposes. With the advancement in the Internet of Things, new research directions are paving the way in serious games. However, the internet connectivity of players in Internetof-things-enabled serious games is a matter of concern and has been worth investigating. Different studies on topologies, frameworks, and architecture of communication technologies are conducted to integrate them with serious games on machine and network levels. However, the Internet of things, whose core requirement is the provision of connectivity on the application layer, has different challenges for more dynamic applications such as serious games. The performance of Internet-of-things-enabled serious games depends on the type of infrastructure (wired, wireless) network and Mobile Ad-hoc Network (MANET) and is subtly different from one type of network to another. This paper investigates the connectivity challenges in the Internet-of-thingsenabled serious games using the mentioned infrastructure and identifies the core requirements for these games. It also aims to evaluate various parameters such as reliability, scalability, response time, to name a few, with varying infrastructure and network types. Results highlight the preliminary infrastructure finding and highlight the core setup for which the games are deployed. Moreover, this work will be a steppingstone for architecting the connectivity in serious games in a typical smart space with many infrastructures such as wired networks, wireless networks, and MANET.
\end{abstract}

Keywords: Internet of things; games AI; serious games; topologies; connectivity

\section{Introduction}

Computer Games are a crucial part of human life which help them to spend their leisure time, generate new ideas and elevate their intellectual level. However, the objective of some games is beyond entertainment, and instead, they are designed with a serious job in mind, such as education, healthcare, 
and training. In literature, it is known as serious games [1]. Serious games are a playful way to spend a healthy life, excitingly educate kids, and joyfully gamify training activities. Such games enable people to concentrate and participate more interactively within the society and helps in nurturing the education system with state-of-the-art methods. Serious games are primarily made for nonentertainment purposes through user interaction and are helpful in education, the healthcare system, training, military, industry, and research. Serious Games and Gamification provide multiple solutions, especially in research and industry.

The recent trend in research is to utilize Internet-of-Things (IoT) [2] with connected sensors to fetch the contextual data from the physical environment and with machine learning, and gamification $[3,4]$ allow players a better insight and an informed experience throughout the lifecycle of the gameplay. One of the goals of this work is to investigate the relationship between IoT and serious games. Simulation of serious games is getting popularity in almost every field of life like military, education [5], industry [6], and medical environment [7] to train and facilitate people for specific conditions. IoT promises the interconnectivity of more networking devices, sensors, technology, data-driven applications, and new software, and it could not be achieved without advancement in the field of IoT [8]. IoT is a network of interconnected devices, sensors, and services to operate smart spaces with virtual identification [9].

The combination of IoT and serious games is a popular research topic and getting attention from academia and industries due to its wide-range implications. The literature for serious games span around frameworks [10], topologies [11], architectures, and applications [12], as shown in Fig. 1. In addition, IoT-enabled serious games have a subtle presence in the literature. Nonetheless, quite a few studies are devoted to playing serious games with IoT to perform a helpful job in smart spaces.

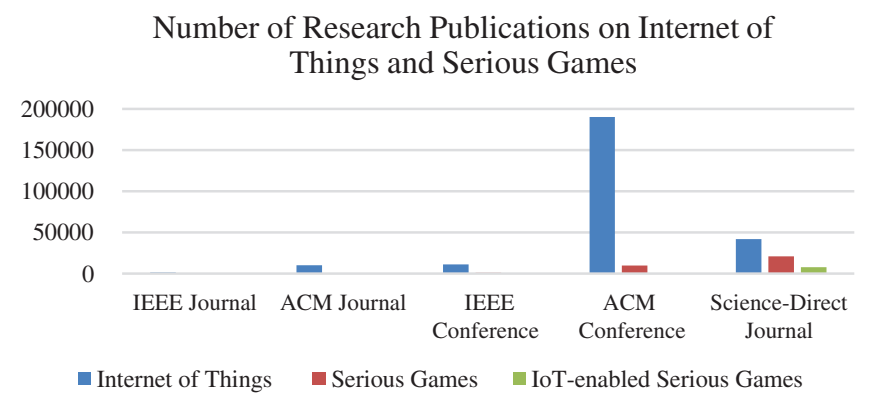

Figure 1: Current trend in literature related to IoT, serious Games, and IoT-enabled games

It is envisioned that one of the core steps in the realization of smart and sustainable cities is to involve the citizens and stakeholders and encourage them to perform valuable activities using gamification and contests. For instance, ranking citizens and municipality members in a smart waste management system can instill a sense of competition. Thus, they can joyfully do their job contributing to the cause of cleanliness without a hassle. Such techniques are used in many different areas, such as smart energy systems, smart health, to name a few. Nevertheless, serious games in the IoT environment warrant connectivity challenges due to the real-time compliance of many games. This paper addresses this challenge and simulates various connectivity infrastructures by varying the number of players and foreseeing its response. The connectivity can be through a wired network, wireless network, or MANET. In wired networks, Internet-of-things-enabled serious games read the data from the surrounding sensors and network devices very reliable without any interruption. However, the reliability in packet delivery and efficiency in terms of battery capacity decreases in a 
wireless network and further decreases the performance in MANET. The final aim of this paper is to highlight the issues related to serious games using IoT in a wired network, wireless network, and MANET.

The rest of the paper contains the following section. In Section 2, related work related to serious games and IoT and vital contributions in these games' architecture, topologies, and frameworks. Section 3 introduces a standardized framework for serious games in IoT and highlights its main components. Section 4 overviews the previous topologies and relates them to IoT-based networks. Section 5 lists the core requirements of IoT-enabled serious games and identifies crucial parameters evaluated on various network infrastructure in Section 6. Finally, Section 7 concludes the paper and highlights potential research directions.

\section{Related Work}

A wide-ranging search has been conducted by investigating related articles from well-known academic databases like ACM, IEEE Xplore, Science Direct and Springer, to mention a few. Some important key search terms are IoT, Serious Games and IoT-enabled serious games. In addition, there is a vast amount of literature surrounding serious game methodologies and frameworks, including surveys of such publications. This section provides the identified research pieces that illustrate some form of modular framework for serious games. For instance, S. Tang and M. Hanneghan [13] identified a model-driven framework for serious games and encouraged the development of serious games for educational purposes by multi-discipline domains. Their framework is modular and therefore encourages adaptation for service-specific applications. Similarly, Lameras et al. [14] introduced a framework for Serious Games for scientific pedagogy use. Their framework focuses on coherently merging the attributes of pedagogy and games to produce better educational games. They also outline a detailed and practical framework for use in such games, elements of which can be applied to eventdriven games in an academic setting. In another study, Koberid et al. [15] presented a framework for achieving flow in educational games. Their work links educational theory with game design and validates to maintaining engagement in educational games. Their research is service-specific and provides additional points for consideration when defining a framework for smart serious games.

Cowley et al. [16] published a novel approach to serious game design by introducing smaller game elements into a framework instead of constructing service-specific modules. This approach leads to creating a modular framework where development can start at any stage based on the principle developed. This modular approach coexists in our solution; however, we focus on producing a modular framework for integrating IoT and Serious Games, which can be utilized to accelerate the production of such applications and further the surrounding academic field.

Research into serious games for obesity by Hassan et al. [17] produced a framework capable of obtaining real-time sensor information from Body Sensor Networks (BSNs) that feed into the game and suggest improvements directly to the players regarding exercise and nutrition. The research mentioned above also outlines the technologies required for a game labeled as pervasive but can be considered for IoT. We extend this type of research by defining an application-neutral topology with a modular outline that will aid researchers in developing service-specific Serious Games using IoT.

Due to IoT's physical and networking nature, topologies are vital for standardization of frameworks for IoT-based applications. We propose a topology for the application area in this research, detailed in Section 4, in the context of current literature [10,11] on game and IoT topologies for the development of IoT-enabled Serious games. A plethora of research exists on service-specific topologies for IoT. In addition, Game engines play a pivotal role in defining serious games in any environment. 
For instance, Unreal Engine, Unity, and DICE are some of the worth-notables [18]. Unreal Engine is used for its extensive tools and features, whereas DICEs Frostbite Engine is widely utilized for Electronic Arts games such as Battlefield. Similarly, Microsoft XNA Game Studio is utilized for the development of Xbox and P.C. games through C\# and Visual Studio and Unity, primarily known for its ease of use and cross-platform game deployment. A game engine could be essential to develop and deploy a Serious Game that connects with IoT, dependent on the assets and virtual environment.

Serious games refer to the use of game techniques or games technology for non-entertainment purposes. Applications include games for health, advertisement, training, education, science, research, production, and work, in which games technologies are used specifically for improving accessibility of simulations, modeling environments, visualization, interfaces, delivery of messages, learning and training, and productive activities such as authoring, development or production [19]. Notable examples of serious games applications include Foldit, a protein-folding game that crowd-sources gamers to help solve real-life problems [20]. To the best of the authors' knowledge, this is the first attempt to investigate the connectivity of players in a serious game using IoT.

\section{Serious Games Standardized Frameworks in the Internet of Things}

The framework for combining IoT and serious games is explaining in this section. This framework can be adjusted according to their research and industry needs $[4,10]$. While designing a framework, many vital aspects of IoT and serious games such as scalability, neutral topology, and neutral applications should be considered. In serious games, scalability is an essential factor because the players can increase drastically, depending on the application's popularity. Some games allocate fewer resources at the server end, and when more players join in the peak time, it is tough to allocate the resource equally to each player at different locations. The situation even worsens in IoT-enabled serious games because new sensors and nodes also need to be accommodated within the same framework. Hence a scalable network is required for serious games [11].

Topology is the crucial element of integration between serious games and IoT. The topology defines the networking requirement for serious games and IoT, and the framework depends on it. However, integrating different technology discourages a single topology as it will be limited for specific services, and the framework could not be used efficiently. Therefore, a multiple topology framework is helpful for numerous users and from multiple locations.

Finally, applications should not be service-specific because of the use of wired, wireless, and MANET. Each network needs different requirements for software as well as hardware. For example, in a wired network, a data-intensive application can be deployed using any wired network, but the same application will not be suited in wireless networks and MANET. Therefore, an application should be developed according to the framework [21].

Based on the following requirements, a framework is designed in a top-bottom approach. The data flow is from the application layer to the middleware layer, and then the data flow back down and updating the state of the game. As a result, a data flow loop will form, which allows the game's progress based on the user's input. For example, if the user completes one stage in the game, then it is allowed to play the next stage of the game, but this progress depends on the condition, which is the completion of the previous phase, and hence the game is updating towards the next stage. The framework comprises the sensing layer, networking layer, middleware layer, data processing layer, and finally, the application layer, as shown in Fig. 2. 


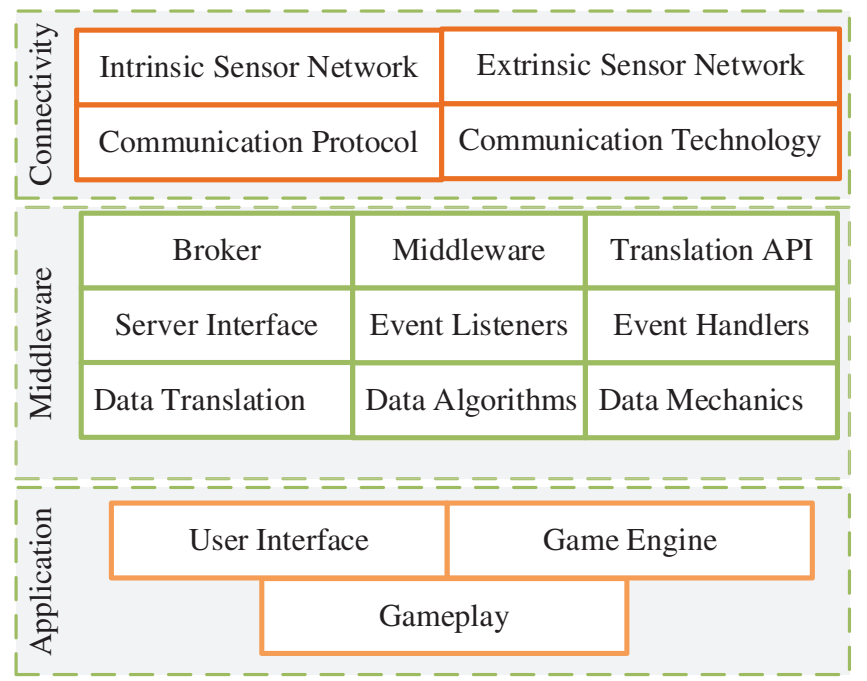

Figure 2: Serious games standard layered infrastructure

a) Sensing is the first layer of the framework, and it is composed of two modules, i.e., extrinsic and intrinsic network. The former defines the physical network that is developed for an application. While the latter represents RFID, human body sensor network, Bluetooth, and Wi-Fi. The extrinsic network represents APIs like traffic, behavior, weather, etc., and applications can use these modules according to their requirements and conditions.

b) The networking is the second layer of the framework, and it is composed of all necessary communication technologies modules to permit the flow of data to the middleware layer. These communication modules allow data communication and networking over wired and wireless networks like WLAN, WPAN, and WWAN [21]. In similar cases, 3G, 4G, and 5G can also be included for the proper operation of the game because it needs internet connectivity. Finally, the communication protocol module represents the underlying protocols used for networking because the application accesses the networking layer to communicate with the middleware layer.

c) The middleware layer is a bridge between all layers and acts as an endpoint for data streaming. In the middleware layer, a broker module handles messages directly transmitted from the sensor network to the server and can be changed according to the application's needs. The data event module is vital in the middleware layer and used for notification of games, point allocation, to name a few, according to the requirement of the application. It is the backbone for the integration of serious games with IoT.

d) The middleware layer contains the data processing layer, and it is composed of a data translation module, data algorithm module, and game mechanic module. The middleware layer is used to manage raw sensor data and other forms of data during gameplay. It contains search engines, data mining, construction of data centers, and intelligent decision [22]. All three modules are essential for serious games applications, especially the translation module, which translated the raw sensors data into an understandable form that can be used in the game. For example, during a game, a Bluetooth network detects the player's presence at a specific time and date and then translates it into the game points for the player as a reward for the presence at a specific time and date. At this stage, the data algorithm module accumulates all these points during the game. Finally, the game mechanic module is activated by directly interacting with 
data translation and triggers a reaction in the gamer like increasing the level of the game, giving bonuses or credits, and unlocking achievements.

e) The application layer is the last layer of the framework, and it offers an interface for user interaction and is known as the user interface module and the game engine. This module defines the game, and both serious games and gamification can be assisted from it because it is used for integration with the IoT. However, the interface module can be removed in some low-level games, like playing with the buzzers. The buzzer game depends on the physical location and instructs the application to react according to the position. Hence, the application layer depends on the kind of application and can be included in the heart of the application/ game [23].

\section{Internet-of-Things-enabled Serious Games Network Topologies}

Integrating IoT and serious games are more pervasive and shifting gaming from a computergenerated environment to a physical environment. Through pervasive games [24], computer games are played with the help of receptive technology. To introduce pervasive behavior in serious games, the user input is considered less intrusive, permitting the user to concentrate on gameplay for the better involvement of the player. Thus, the serious games have a positive impact on the behavior of the player. This positive impact on behavior can be achieved in a learning environment. Following topologies are traditional but applied to the modern setting such as IoT in the same manner with minor modifications.

\subsection{Client Server Topology}

For online gaming, the services provided by the client-server architecture [25] are fundamental for developing serious games using IoT. In this approach, the player/user needs services from the server, and it depends on the server's response, reply, services, and behavior. In this approach, the player cannot communicate directly with another player but through the server. The main limitation in clientserver topology is the central administration system. If the server failure occurs due to hardware fault, software fault, or human error, then the whole network will not function, and all the activities are stopped until the replacement or repairing of the server. Similarly, scalability is also a big concern for client-server topology, especially in wireless and MANET infrastructure.

\subsection{Peer to Peer Topology}

To overcome the issues of scalability and central point of failure, a newer approach known as peerto-peer topology [26] is preferred for large multiplayer online groups. This topology is not limited to centralized administration, and every player can play with another player and facilitate other players during the game. This will distribute the load from the network on every player, and if there is a failure of one user due to any hardware or software fault, the games continue without affecting the whole network and hence can be termed as reliable. This approach is also very scalable in the wired network due to the availability of hardware and other resources, but it is not very scalable in wireless and MANET. The work done in [27] known as MOPAR proposed a hybrid solution to increase the scalability and fault tolerance in serious gaming. The results of MOPAR are worth investigating, especially in the mobile environment.

\subsection{Cloud Topology}

The cloud topology forms two tiers. Tier 1 designed the cloud for public services, and tier 2 composed servers nearer to the player. It is a preferable solution for online gaming regarding scalability 
and decentralized administration but at the cost of higher price and security. The price is higher for endusers, and the security of the cloud is also coming into question due to public access. The cloud-centric [28] idea is getting a lot of reputation in serious gaming using IoT and other private and middleware cloud competing paradigms. It is instrumental in a large-scale network for a massive number of players with multiple applications. However, for the small infrastructure which can be operated from a local server having a small number of players and IoT devices, the use of the cloud can be overkill [29].

A hybrid topology can be utilized without a sink node but only involve users, servers, and sensors. For serious games, the sink is used for the connectivity of the Internet, which has fewer resources and is placed at the edge of the network. However, in the absence of the sink node, the player will be relying on the game requirement and power management. The limitation of this hybrid approach is that the number of players on the edge of the network is not entertained, and those nodes which have no access to the power will also be excluded from the gameplay.

\subsection{Infrastructure-Less Topology}

This topology is divided into wireless networks and MANET. A wireless network is a combination of wired infrastructure along with wireless connectivity. For example, a number of players are connected wireless with an access point, Wi-Fi and router. On the other hand, in MANET, all the nodes should be interconnected wirelessly without any infrastructure. To use wireless networks and MANET in an IoT along with serious game integration is a challenging task. However, the work done in [30] discussed two techniques to mitigate this challenge, i.e., stack-based and topology-based integration. The orientation of players and computing resources in an IoT-enabled serious game can be placed in conventional client-server, peer-to-peer, cloud, and MANET, as shown in Fig. 3.

Furthermore, two approaches like hybrid and access point are suggested in topology-based integration. In the former approach, the player can play through the Internet with intelligence and redundancy, and freedom. However, in the latter method, the player depends on the sink node for intent connectivity because nodes in the edge in the wireless less and especially in MANET have fewer resources to receive the real-time data due to frequent topology changes and mobility of the player. Therefore, it is a very tedious task to develop a scalable infrastructure-less network that can interact with the virtual and physical environment in the presence of a sink, multiple standards, and vendors.

To resolve the sink node issue and make the infrastructure-less topology more efficient, reliable, and scalable, a weighted network topology is introduced that shows the sink nodes and sensors with edge weight [31]. Due to the introduction of this approach, sinkholes are avoided in the network, and the scale of the network also increases with the maximum lifetime of the nodes as well as the network. Hence a large amount of data can be used by the player with a maximum number of users.

\section{Requirements for Internet-of-Things-Enabled Serious Games Topologies}

To design a topology for Serious Games, the topology of the IoT should be considered. IoT combines a wired network, wireless network, MANET, and wireless sensor network (WSN). The context data of the sensor between the player and the server or between players themselves are also worth considering for forming an IoT-aware topology. Following are the parameters that are crucial for the connectivity of players and IoT components and must be considered during the formation of the topology. 
(a)

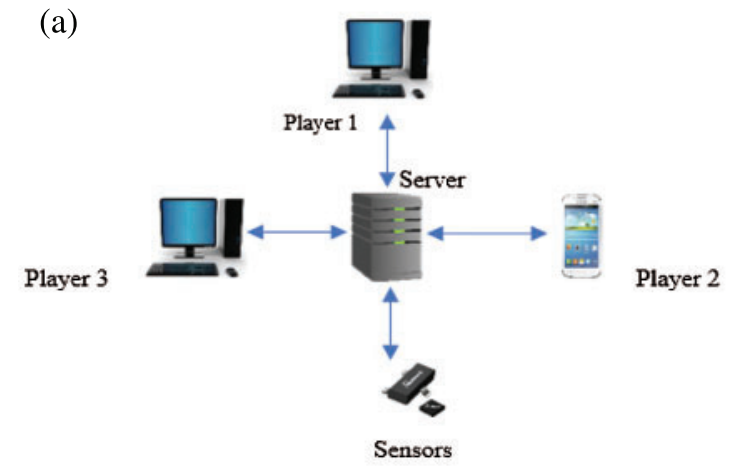

(c)

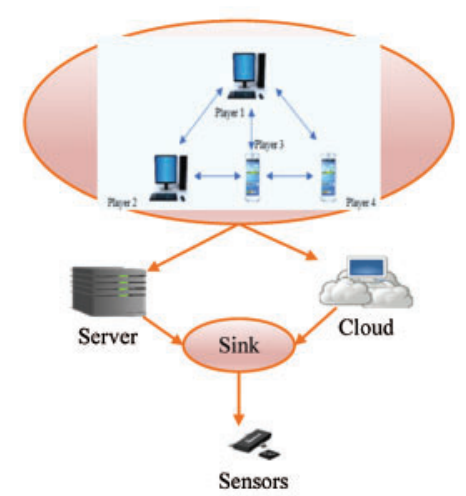

(b)

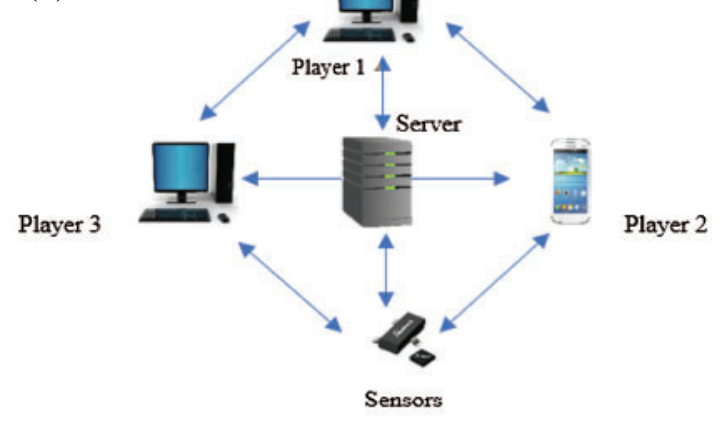

(d)

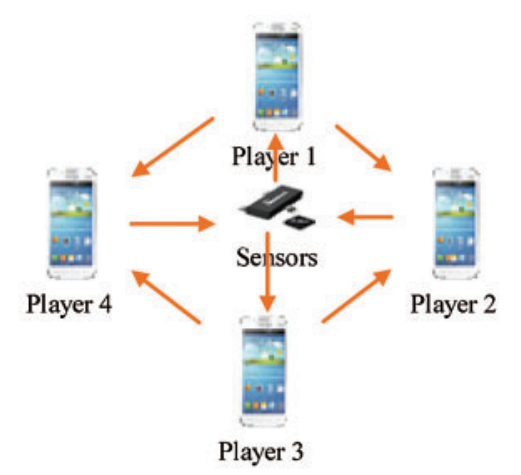

Figure 3: State-of-the-art topologies for connectivity in IoT-enabled serious games. (a) Client-Server topology (b) Peer-to-peer topology (c) Cloud topology (d) MANET topology

\subsection{Scalability}

As mentioned in earlier sections, scalability is one of the most vital and core attributes of games. It is a fundamental factor for designing any topology and application. In IoT-enabled Serious Games, the attributes of WSN, MANET, and wireless networks should be considered. All the data either through MANET or wireless network must be communicated through sensors directly or indirectly. The data transmission and reception of WSN are different from the wired network, wireless network, and MANET because the player's lifetime is faded sooner in MANET than a wired network, wireless network.

Similarly, the player's lifetime is completed sooner in a wireless network than in a wired network. But, on the other hand, the scalability increases by connecting multiple players from MANET compared to wired and wireless networks. Similarly, the scalability of the wireless network increases as compared to a wired network. Therefore, a scalable topology in Serious Games should allow the players to be added continuously without significant software changes irrespective of the number of the players.

\subsection{Lifetime of the Player}

It is another crucial factor, and it should be less utilized for the network to work efficiently. In serious games, for those players that are not operational, then their sleep modes should be functioning at that specific time, which will decrease the energy utilization of the player and the network and hence 
increase the lifetime of the player. The player's energy in MANETs depletes sooner in MANET and wireless networks than in a wired network. It affects the sensory demands of a Serious Games topology during the peak time and bandwidth within a wireless network and MANET.

\subsection{Reliability}

Some applications of Serious Games require high processing, large files, and bandwidth. Hence, those players operating from wired networks offer more reliability than wireless networks and MANET due to the high demand for data from a specific application. In addition, the wired network is more reliable due to the availability of power resources, large memory, increased processing speed, and high software and hardware quality compared to wireless networks and MANET.

\subsection{Efficiency}

In Serious Games, delay efficiency affects the player's performance because the player needs the data in real-time. Also, priority-based decisions are significant, and it is near-impossible during peak time and with a maximum number of players in wireless networks and MANET.

\subsection{Security}

Security from the network perspective is essential in serious games because the sensors are connected with the Internet using protocols and encryption techniques, which preserve the valuable data of the player by avoiding harmful attacks. However, due to the sensors' small size and low processing capabilities, the sensors use lightweight security protocols and are therefore very vulnerable to such attacks. Also, the player in MANET and wireless networks are vulnerable to malicious attacks. On the other hand, players within a wired network have less vulnerability to malicious attacks due to high processing and high memory.

\section{Performance Evaluation}

The CoAP [32] protocol is implemented in a Network Simulator 2 (NS2). The Ubuntu Platform of NS 2.35 is utilizing C++ and OTCL/TCL as Back and front-end, respectively. Multiple AWK scripts are developed to collect data from trace files with the help of a BASH script. The parameters of simulations are given in Tab. 1.

Table 1: Parameters of NS-2 simulator

\begin{tabular}{ll}
\hline Compared protocols & CoAP \\
\hline Quantity of nodes & 50 \\
Covered simulation area & $1000 * 1000$ \\
Time of simulation & $450 \mathrm{secs}$ \\
Packet size & $512 \mathrm{bytes}$ \\
Maximum speed & $5 \mathrm{~m} / \mathrm{s}$ \\
\hline
\end{tabular}

A random way-point mobility model is used for different network scenarios and is implemented to evaluate the performance. Pause time in this simulation shows with zero seconds gives continuous mobility, and this pause time increases up to 1000 seconds until the simulation runs for 450 seconds. 
A CoAP protocol is used in a wired network, wireless network, and MANET in this work. A Wired, wireless and MANET network compares player performance in terms of scalability, reliability, efficiency, network lifetime, and security. The performance graph of each parameter is shown in Fig. 4. The discussion on each of them is outlined in the subsequent sections.

\subsection{Scalability}

As elucidated in Fig. 4a, the scalability in a wired network is limited to a specific range because the deployment of wires is limited to certain coverage areas like health centers, gyms, or colleges. Therefore, the scalability drastically drops from 1200 meters in the wired network and the wireless-network is falling from 1700 meters because we assumed this range in the simulation. On the other hand, the scalability of wireless networks is increasing compared to wired networks because the number of simulation areas is limited to the hotspot. On the other hand, the scalability in MANET increases with the growing number of players due to no limitations of area and range.
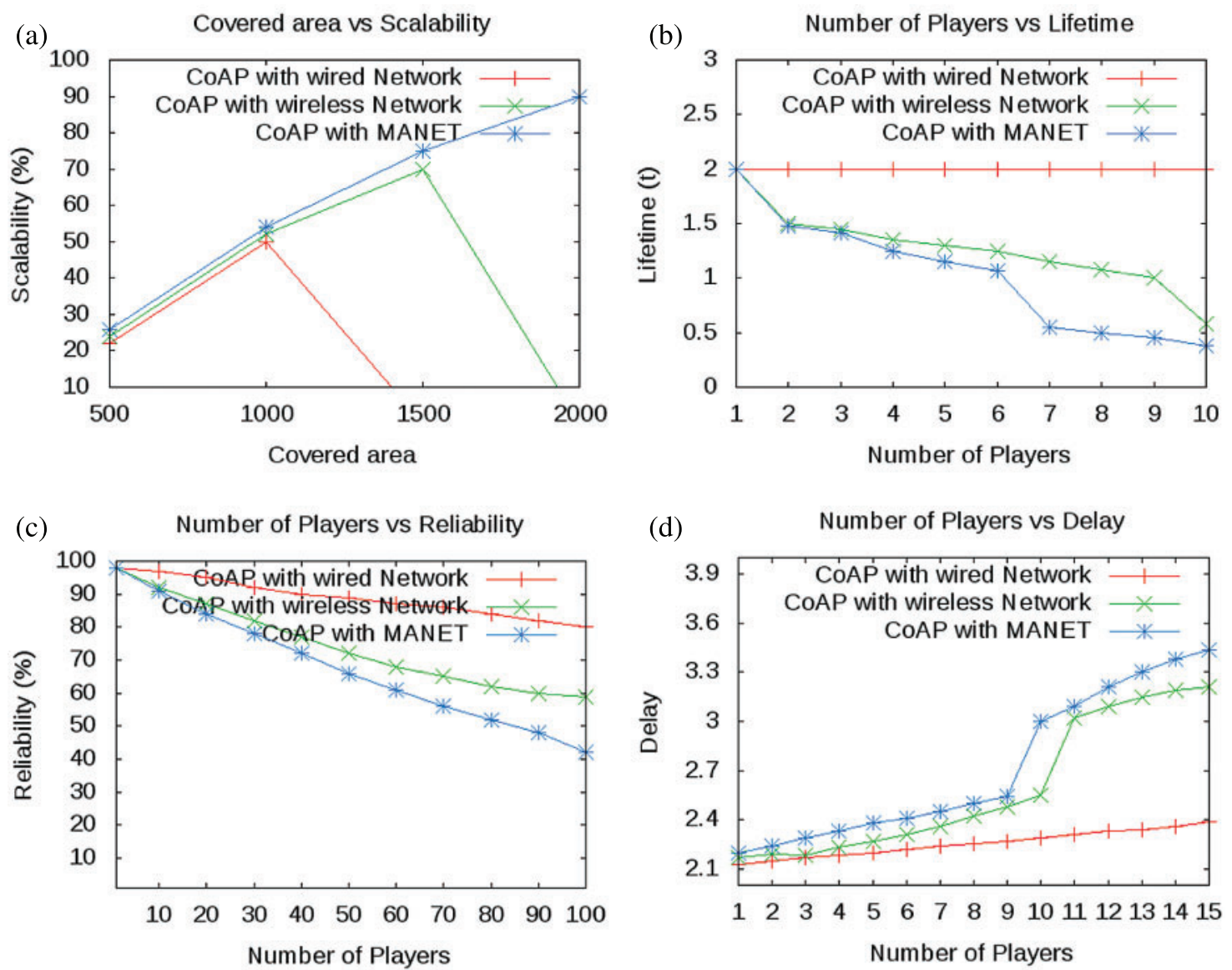

Figure 4: Performance evaluation in terms of different parameters. (a) Scalability (b) Players' lifetime (c) Reliability (d) Delay 


\subsection{Lifetime of the Player}

The lifetime of a player is assessed with the amount of time the player keeps connected with the network. Once more and more players join the game, at times, a bottleneck situation occurs and the players got disconnected from the network. The simulation of players connectivity time with respect to the number of players is carried out. As exhibited in Fig. 4b, the player's lifetime in a wired network is uniform, and the player is playing the games without any disruption due to the continuous supply of power. In the simulation, it is assumed that the player initially has a battery capacity of two hours. In the wireless network, the player has allowed the same time within the power supply range, but sometimes it is out of the reach of the power supply, leading to a subtle degradation of the player's life over time. On the other hand, the player's lifetime in MANET decreases drastically compared to wired and wireless networks due to the absence of a power supply.

\subsection{Reliability}

The reliability of the game depends on the connectivity time of the players and the quality of service. In this work, reliability is computed as the percentage number of packets reached. The network packet is the payload of the CoAP commands, which is one of the popular connectivity protocols in the IoT domain. As depicted in Fig. 3c, the player's reliability in a wired network increases due to the enormous bandwidth of the network. This colossal bandwidth enables the player to receive massive data in real-time and thus increases reliability $[33,34]$. In a wired network, the packet drop decreases due to wired infrastructure and resources compared to wireless networks and MANET. On the other hand, due to decentralized infrastructure, topology changes, and wireless medium, the packet drop increases in the wireless network and MANET and decreases the reliability compared to the wired network.

\subsection{Efficiency}

Efficiency is computed with respect to the delays in the network. The delay with the increasing number of players is recorded in milliseconds. As portrayed in Fig. 3d, the player's efficiency in the wireless network decreases due to lesser- available bandwidth and packet drop, but it is significantly better compared to MANET. The packet drop is more in MANET than the wireless network and wired network and therefore increases the delay. The increase in the delay comes at the cost of efficiency in MANET compared to wired and wireless networks. The efficiency in MANET is significantly lower than wired and wireless networks due to the less bandwidth availability. Also, the performance is further decreased in real-time scenarios due to frequent packet drops. Hence, reduces the efficiency in term of delay.

\section{Conclusion}

The literature on IoT-enabled Serious Games is still nascent, yet it has a tremendous potential unearthed in upcoming studies. One of the core attributes these games is the connectivity challenge in the application domain $[33,34]$. A variety of research is conducted on the topology of serious games, but there is a considerable gap in IoT-enabled serious games. The network topology plays a vital role in serious games, and it improves their performance and also the player's performance. Three types of topologies, namely wired network, wireless network, and MANET are discussed in this paper. Their impact on the player and the serious game itself is evaluated with CoAP as a connectivity protocol in an IoT environment [28]. Four different parameters, namely scalability, reliability, efficiency, and player's lifetime, are assessed and compared on the different topologies, and the results are obtained. 
According to the results, the scalability of MANET and wireless network was better than the wired network. However, the reliability, efficiency, network lifetime, and security are better in wired networks than wireless networks and MANET.

Similarly, the reliability, efficiency, and players' lifetime are better in a wireless network than MANET. From the results, it is evident that the connectivity in serious games with IoT should consider all the factors and go for the best compromise. The future work of this research is to propose a hybrid topology that integrates the positives of all the above three topologies and re-simulates it with more protocols such as HTTP, MQTT, and CoAP. The intelligence in the network layer can also be a factor worth-investigating in the serious games domain.

Funding Statement: This research is supported by the Ministry of Culture, Sports and Tourism and Korea Creative Content Agency (Project Number: R2020040243) and by the National Research Foundation of Korea (NRF) Grant funded by the Korean government under Grant NRF2021R1I1A1A01045177.

Conflicts of Interest: The authors declare that they have no conflicts of interest to report regarding the present study.

\section{References}

[1] C. Girard, J. Ecalle and A. Magnan, "Serious games as new educational tools: How effective are they? A meta-analysis of recent studies," Journal of Computer Assisted Learning, vol. 29, no. 3, pp. 207-219, 2013.

[2] J. Gubbi, R. Buyya, S. Marusic and M. Palaniswami, "Internet of Things (IoT): A vision, architectural elements, and future directions," Future Generation Computer Systems, vol. 29, no. 7, pp. 1645-1660, 2013.

[3] S. Kumar, "Bridging the gap between the real world and video games using iot," B.S. Thesis, School of computer science and engineering, Galgotias university, Greater Noida, Uttar Pradesh, India, 2020.

[4] H. Y. Kim, "A design and implementation of a framework for games in IoT," Journal of Supercomputing, vol. 74, no. 12, pp. 6516-6528, 2018.

[5] H. John, S. Tang, S. Mukhopadhyay and M. H. Yap, "A randomised control trial for measuring student engagement through the internet of things and serious games," Internet of Things, vol. 13, no. 15, pp. 100332, 2021.

[6] D. Sharma, J. C. Lakhmi, M. Favorskaya and R. J. Howlett, Fusion of smart, multimedia and computer gaming technologies, 1st ed., vol. 1. New York, United States: Springer International Publishing, 2015.

[7] E. I. Konstantinidis, "The interplay between IoT and serious games towards personalised healthcare," in 9th Int. Conf. on Virtual Worlds and Games for Serious Applications, Athens, Greece, pp. 249-252, 2017.

[8] S. Ahmad, I. Hussain, M. Fayaz and D. Kim, "A distributed approach towards improved dissemination protocol for smooth handover in mediasense IoT platform," Processes, vol. 6, no. 5, pp. 46-61, 2018.

[9] S. Ahmad and D. Kim, "A multi-device multi-tasks management and orchestration architecture for the design of enterprise IoT applications," Future Generation Computer Systems, vol. 106, pp. 482-500, 2020.

[10] J. Henry, S. Tang, M. Hanneghan and C. Carter, "A framework for the integration of Serious Games and the Internet of Things (IoT)," in 2018 IEEE 6th Int. Conf. on Serious Games and Applications for Health (SeGAH), Vienna, Austria, IEEE, pp. 1-8, 2018.

[11] J. Melthis, S. Tang, P. Yang, M. Hanneghan and C. Carter, "Topologies for combining the internet of things and Serious Games,” Journal of Intelligent \& Fuzzy Systems, vol. 31, no. 5, pp. 2685-2696, 2016.

[12] C. Garcia, F. T. Saenz, F. G. Burgos and A. F. Skarmeta, "Integration of Serious Games and IoT data management platforms to motivate behavioural change for energy efficient lifestyles," in Proc. on Global Internet of Things Summit, Dublin, Ireland, 2017. 
[13] S. Tang and M. Hanneghan, "A model-driven framework to support development of serious games for game-based learning," in 2010 Int. Conf. on Developments in eSystems Engineering (DESE, London, UK, pp. 95-100, 2010.

[14] P. Lameras, A. Sylvester, De. Sara, P. Panagiotis and D. Ian, "Science teachers' experiences of inquiry-based learning through a serious game: A phenomenographic perspective," Smart Learning Environments, vol. 8, no. 1, pp. 633, 2021.

[15] S. E. Koberid, G. Woodid, K. Killi, K. Moeller and M. Ninaus, "Game-based learning environments affect frontal brain activity," PLOS One, vol. 15, no. 11, pp. e0242573, 2020.

[16] B. U. Cowley and C. Bateman, "Green my place: Evaluation of a serious social online game designed to promote energy efficient behaviour change," International Journal of Serious Games, vol. 4, no. 4, pp. 71-90, 2017.

[17] M. M. Hassan, M. S. Hossain, A. Alamri, M. A. Hossain, M. A. Qureshi et al., "A cloud-based serious games framework for obesity," in Proc. of the 1st ACM Multimedia Int. Workshop on Cloud-Based Multimedia Applications and Services for e-Health, Nara, Japan, pp. 15-20, 2012.

[18] J. Gregory, "Game engine survey," Game engine architecture, 3rd ed., Florida, US: CRC Press, ch. 1, sec. 5, pp. 33-40, 2018.

[19] F. Laamarti, M. Eid and A. El Saddik, "An overview of serious games," Int. Journal of Computer Games Technology, vol. 2014, no. 3, pp. 1-15, 2014.

[20] V. Curtis, "Motivation to participate in an online citizen science game: A study of Foldit," Science Communication, vol. 37, no. 6, pp. 723-746, 2015.

[21] D. Guinard, S. Karnouskos, V. Trifa, B. Dober, P. Spiess et al., "Interacting with the SOA - based internet of things: discovery, query, selection, and on-demand provisioning of web services," IEEE Transactions on Services Computing, vol. 3, no. 3, pp. 223-235, 2010.

[22] B. Xu, L. D. Xu, H. Cai, C. Xie, J. Hu et al., "Ubiquitous data accessing method in IoT-based information system for emergency medical services," IEEE Transactions on Industrial Informatics, vol. 10, no. 2, pp. 1578-1586, 2014.

[23] S. Fang, L. Xu, H. Pei, Y. Liu, Z. Liu et al., "An integrated approach to snowmelt flood forecasting in water resource management," IEEE Transactions on Industrial Informatics, vol. 10, no. 1, pp. 548-558, 2014.

[24] T. H. Laine and C. I. Sedano, "Distributed pervasive worlds: The case of exergames," Journal of Educational Technology \& Society, vol. 18, no. 1, pp. 50-66, 2015.

[25] A. B. Sallow, H. I. Dino, Z. S. Ageed, M. R. Mahmood and M. B. Abdulrazaq, "Client/Server remote control administration system: Design and implementation," Int. Journal of Multidisciplinary Research and Publications, vol. 3, no. 2, pp. 5-11, 2020.

[26] V. V. Kumar, D. Mani, P. V. Raja, P. Kanmani, V. Priya et al., "Design of peer-to-peer protocol with sensible and secure IoT communication for future internet architecture," Microprocessors and Microsystems, vol. 78, pp. 103216, 2020.

[27] A. P. Yu and S. T. Vuong, "MOPAR : A mobile peer-to-peer qverlay architecture for interest management of massively multiplayer online games," 15th Int. Workshop on Network and Operating System Support for Digital Audio and Video. Washington, USA, 99-104, 2005.

[28] S. Ahmad, L. Hang and D. Kim, "Design and implementation of cloud-centric configuration repository for DIY IoT applications," Sensors, vol. 18, no. 2, pp. 474-494, 2018.

[29] D. Mishra, M. El Zarki, A. Erbad, C. H. Hsu, N. Venkatasubramanian et al., "Clouds + Games: A multifaceted approach," IEEE Internet Computing, vol. 18, no. 3, pp. 20-27, 2014.

[30] C. Alcaraz, P. Najera, J. Lopez and R. Roman, "Wireless sensor networks and the Internet of Things : Do we need a complete integration?," 1st Int. Workshop on the Security of the Internet of Things (SecIoT"10). Tokyo, Japan, 1-8, 2010.

[31] D. G. Zhang, Y. N. Zhu, C. P. Zhao and W. Dai, "A new constructing approach for a weighted topology of wireless sensor networks based on local-world theory for the Internet of Things (IOT)," Computers \& Mathematics with Applications, vol. 64, no. 5, pp. 1044-1055, 2012. 
[32] K. Kuladinithi, O. Bergmann, T. Ptsch, M. Becker and C. George, "Implementation of CoAP and its application in transport logistics," in proc. of the Workshop on Extending the Internet to Low power and Lossy Networks, Chicago, USA, 2011.

[33] S. Ahmad, A. Khudoyberdiev and D. Kim, "Towards the task-level optimal orchestration mechanism in multi-device multi-task architecture for mission-critical IoT applications," IEEE Access, vol. 7, no. 1, pp. 140922-140935, 2019.

[34] S. Ahmad, S. Malik, I. Ullah, D. H. Park, K. Kim et al., "Towards the design of a formal verification and evaluation tool of real-time tasks scheduling of IoT applications," Sustainability, vol. 11, no. 1, pp. 204-226, 2019. 